\title{
Nachdiplomkurs «Wirksam managen im Gesundheitssystem»
}

Spitäler und andere Organisationen des Gesundheitswesens weisen einen zunehmenden Führungsbedarf auf. Management wird immer wichtiger. Aber es braucht nicht irgendein Management, sondern einen integrativen Ansatz. Die Fragen, wie konkrete Organisationseinheiten mit ihren Aufgaben, Strukturen und Personen lebensfähig positioniert, gestaltet und optimiert werden können, stellen sich mit Nachdruck. Managementkompetenz ist darum essentiell. Führungskräfte aller Berufsgruppen benötigen die entsprechenden spezifischen Kenntnisse und Fähigkeiten. Unser Weiterbildungsprogramm «Wirksam managen im Gesundheitssystem» vermittelt Ihnen genau dies.

\section{Themen und Lernziele}

\section{Element 1: Gestalten}

Die Wirksamkeit von Führung; Führung und Vielfalt; Herausforderungen und Antworten; Managementkonzepte; integratives Management; Systemisches Denken und Handeln als «key» im Umgang mit komplexen Situationen; Aktuelle Ansätze im Gesundheitswesen.

\section{Element 2: Führen}

Klarheit und Entschiedenheit; Kommunikation als Medium; Bedeutung der Multiperspektivität; Kooperative Arbeitsformen in Teams und Organisationen; Zur Bedeutung professioneller Subkulturen.

\section{Element 3: Intervenieren}

Durchführung eines realen Projektes in einem Spital.

\section{Element 4: Wirken}

Projektmanagement; Selbstführung / Coaching; Auftrittskompetenz.

\section{Element 5: Steuern}

Gesundheitsökonomie und -politik; Schwierige Gespräche führen; Organisationen entwickeln.

\section{Element 6: Integrieren}

Die vielfältigen Facetten der Führung; Rückblick und Ausblick.

\section{Daten und Ort}

\section{Element 1}

Mittwoch, 30.8.2006,

bis Freitag, 1.9.2006

Element 2

Montag, 30.10.2006,

bis Mittwoch, 1.11.2006

Element 3

Mittwoch, 29.11.2006,

bis Samstag, 2.12.2006

\section{Element 4}

Mittwoch, 10.1.2007,

bis Freitag, 12.1.2007

\section{Element 5}

Montag, 5.3.2007,

bis Mittwoch, 7.3.2007

Element 6

Montag, 23.4.2007,

bis Dienstag, 24.4.2007

College-M

College-M

Projektort

College-M

College-M

College-M

\section{Programmleiter}

Dr. Christof Schmitz, Corporate Pragmatics, Zürich

Betriebswirt und Soziologe. Seit 1994 mit eigener Firma corporate pragmatics, Zürich, tätig, die sich speziell mit Veränderungsprozessen, der Optimierung der Intelligenz von Organisationen und der Steigerung ihrer Innovativität beschäftigt. Dozent an verschiedenen Universitäten und Fachhochschulen. Co-Leiter des College-M.

\section{PD Dr. med. Peter Berchtold}

Facharzt Innere Medizin. Langjährige Spitalarzttätigkeit und Führungserfahrung auf allen Funktionsebenen. Mitbegründer und seit 1999 Leiter des College für Management im Gesundheitswesen (College-M) in Bern, einer Stiftung der schweizerischen Verbände der Gesundheitsberufe mit den Kernbereichen ManagementTraining, Forschung und Entwicklung.

\section{Kurskosten/Informationen}

Die Kosten betragen Fr. 7950.- und beinhalten alle Programmaktivitäten und Kursunterlagen.

Detaillierte Informationen finden Sie unter: www.college-m.ch oder erhalten Sie durch: College-M, Freiburgstrasse 41, 3010 Bern, Tel. 03163230 26, Fax 03163230 25, E-Mail: info@college-m.ch. 